\title{
Predictions of Allowable Sensor Error Limit for Cycle-Slip Detection
}

\author{
By Junesol Song,${ }^{1)}$ Younsil KIM,${ }^{1)}$ Ho Yun, ${ }^{1)}$ Byungwoon PARK ${ }^{2)}$ and Changdon KeE ${ }^{1)}$ \\ ${ }^{1)}$ School of Mechanical and Aerospace Engineering and the Institute of Advanced Aerospace Technology \\ at Seoul National University, Seoul, South Korea \\ ${ }^{2)}$ Department of Aerospace Engineering, Sejong University, Seoul, South Korea
}

(Received March 14th, 2013)

\begin{abstract}
Cycle-slip detection is mandatory prior to estimating positions based on carrier-phase observations. Recently, inertial sensors are integrated to detect cycle-slips regardless of sizes and combinations of cycle-slips in different frequencies. However, since inertial sensors contain errors such as random noise and bias, performance of the cycle-slip detection depends on the sensor performance. In general, there is a trade-off relationship between cost and performance of sensors, and we need to select appropriate sensors to achieve required cycle-slip detection performance. Therefore, we need a standard to select appropriate sensors. This paper introduces a procedure to predict allowable sensor error limit for gyroscope and odometer aided cycle-slip detection using theoretical formulation. By using error models of sensors, an error equation is set to predict error contributions from the sensors contained in the monitoring value that is used for cycle-slip detection. Simulation data is used to evaluate derived error equation and predict the error limit of sensors achieving objective performance. As a result, allowable sensor error region is predicted to detect 1 cycle-slip as a minimum detectable cycle-slip. This approach can be extended to accelerometers, and then it can be applied not only to vehicles on land, but also to those in aerospace.
\end{abstract}

Key Words: Cycle Slip, MEMS Gyroscope, Odometer, Error Analysis

\section{Nomenclature}

$\phi$ : carrier-phase observation in meter

$\vec{r}$ : position vector

$\vec{h}$ : heading vector

$\theta$ : heading angle

$p$ : odometer output in meter

$M$ : the number of pulses counted by odometer during the vehicle motion

$d$ : uniformly distributed random variable

$g:$ a random variable

$\dot{\theta}$ : angular rate

$S$ : scale factor of gyroscope

$b$ : bias error of gyroscope in $\mathrm{deg} / \mathrm{hr}$

$\varepsilon:$ noise error of gyroscope in $\mathrm{deg} / \mathrm{hr}$

$\sigma:$ standard deviation

$\vec{D}$ : range vector

$D$ : norm of a range vector

$\vec{p}$ : displacement vector

$\delta \vec{D}:$ displacement vector of satellite motion

$\overline{L O S}$ : line-of-sight vector

$\gamma, v:$ angle between two vectors

$\lambda$ : wavelength

ARW: angular random walk in deg $/ \sqrt{\mathrm{hr}}$

BS: bias stability in $\mathrm{deg} / \mathrm{hr}$

$\Delta T$ : time interval for GPS output rate

$f$ : gyroscope output rate in $\mathrm{Hz}$

$\delta(\cdot)$ : time difference operator

$\Delta(\cdot)$ : single difference operator between two stations

$\nabla(\cdot)$ : single difference operator between two satellites
Subscripts

RS: reference station

$\mathrm{U}$ : user

$k: k$-th epoch

$\mathrm{t}$, true: true value

error: error value

max: maximum value

Superscripts

$i, j$, ref: satellite index

\section{Introduction}

Carrier-phase based positioning methods including realtime kinematics (RTK) and network RTK allow centimeterlevel accuracy in their estimates, and are widely used for many applications, such as surveying and geodesy. These days, the demand for precise positioning of kinematic users such as vehicles in land and aerospace based on carrierphase measurements has been growing. For aerospace vehicles, precise positioning can be used for landing. ${ }^{1)}$ In addition, for land vehicles, there are needs for efficient management of traffic control and safety considerations such as automatic driving systems, intelligent transportation systems (ITS) and the monitoring of trucks carrying hazardous materials. Many research studies have been conducted applying carrier-phase based positioning to the land vehicle environment. ${ }^{2,3)}$

Carrier-phase based positioning can provide accurate positioning results; however, they are vulnerable to cycle slips. A cycle slip is an integer jump in the ambiguity of a carrier-phase observable due to an instantaneous loss of 
the phase lock when the GPS signal is blocked by obstructions. If cycle slips are not detected and isolated, they can induce large errors in the position domain, according to the size of the cycle slip and the satellite constellation. Alternatively, it will re-initialize ambiguity resolution.

Many cycle slip detection algorithms have been proposed over the years. Blewitt, ${ }^{4)}$ Gao et al., ${ }^{5,6)}$ Neumann et al. ${ }^{7)}$ and Bisnath and Langley ${ }^{8)}$ proposed algorithms using linear combinations of double differenced observables from dual-frequency receivers. Dai et al. ${ }^{9)}$ used measurements from a multiple-frequency (more than three frequencies) receiver. However, since such algorithms take linear combinations of more than two observations in different frequencies, they cannot detect cycle slips of some special combinations. Those cases include cycle slips occurring in every frequency, as well as the same ratio as that of coefficients for linear combination of multiple observations in different frequencies. In order to detect cycle slips in every case, inertial sensors can be used for cycle slip detection. Therefore, Colombo, ${ }^{10)}$ Altmayer, ${ }^{11)} \mathrm{Du},{ }^{12)}$ Takasu and Yasuda ${ }^{13)}$ integrated GPS/INS to detect cycle slips more precisely. In this aspect, we focus on cycle slip detection using inertial sensors in this paper.

On the other hand, performance of the cycle slip detection depends on errors contained in sensor measurements. There is a wide range of choices for sensors based on the associated error attributes, such as noise and bias. The cost of a sensor and its performance in terms of noise and bias error characteristics are closely related to each other. In order to develop a cost effective system, appropriate sensors whose costs are reasonable and at the same time satisfy desired performance requirements of the algorithm need to be identified before the system is developed and operated. Therefore, we need a standard to decide appropriate sensors; however, there are no previous works on sensor decision. Many other researchers have studied on this topic such as Carlson, ${ }^{14)}$ $\mathrm{Yu}^{15)}$ and Park. ${ }^{16)}$

In this paper, we propose an algorithm to predict allowable sensor error limit for cycle slip detection. First, we introduce a cycle slip detection algorithm to derive error equation. We then formulate an error equation of the monitoring value for cycle slip detection based on error models of external sensors and predict allowable sensor errors of corresponding sensors conservatively. Since odometers are already installed in vehicles, it does not incur any additional cost. Therefore, we choose an odometer and gyroscope for cycle slip detection. If we choose an accelerometer instead of odometer, this cycle slip detection algorithm can also be applied for users in aerospace. After setting an error equation, simulation data are used to evaluate performance of the cycle slip algorithm and check the validity of the derived error equation. The preliminary predictions of sensors for cycle slip detection algorithm using un-difference GPS observations are shown in Ref. 17). In this paper, we extend this method to the cycle slip detection algorithm using double difference GPS measurements and re-formulate error equations. This case provides more reliable results since receiver clock bias is completely eliminated by double difference. Moreover, we further consider additional sensor errors such as bias stability of the gyroscope and scale factor error of the odometer to reflect reality more closely.

\section{Algorithm Formulation and Error Analysis}

\subsection{Cycle slip detection algorithm}

In order to resolve ambiguity, carrier-phase measurements should be handled as double difference. In this process, clock bias terms are completely eliminated and atmosphere related errors and orbit errors are greatly reduced if the user is within the coverage area of the reference station. ${ }^{18)}$ If we take the time difference once more, cycle slip can be shown as a jump error in the final measurement. Therefore, the monitoring value, MV for cycle slip detection can be expressed as the following equation.

$$
M V^{i}(k)=\delta^{i} \nabla^{j}{ }_{\mathrm{U}} \Delta_{\mathrm{RS}} \phi(k)-\delta^{i} \nabla^{j}{ }_{\mathrm{U}} \Delta_{\mathrm{RS}} D(k)
$$

In order to calculate the second term in Eq. (1), positions of the user, reference station and two satellites at the $k$ th and $(k-1)$ th epochs need to be known. Since the navigation data and the coordinate of the reference station are received from satellites and reference station respectively, all positions except for the user at the $k$ th epoch are known. By knowing the carrier-phase based position of the user at the previous epoch, the displacement vector of the user between previous and current epoch can be estimated by using the gyroscope and the odometer sensors. The estimated position of the user at current epoch can be expressed as the following equation.

$$
\hat{\vec{r}}_{\mathrm{user}}(k)=\vec{r}_{\mathrm{user}}(k-1)+\delta p_{\mathrm{t}}(k) \cdot \hat{\vec{h}}(k)
$$

In this equation, $\vec{r}_{\text {user }}(k-1)$ is the carrier-phase based user position at the previous epoch, which is known at the current epoch, $\delta p_{\mathrm{t}}(k)$ is the distance traveled by the user between two successive epochs, which can be determined from the odometer output, and $\hat{\vec{h}}(k)$ is the heading vector estimated from the gyroscope output. With this estimated user position at the current epoch, the rate of double difference range in Eq. (1) can be calculated. Since atmosphere related errors and orbit errors are negligible due to the manner mentioned before, Eq. (1) can be assumed to have only the change in the integer ambiguity; therefore, cycle slip can be detected by monitoring the MV with an appropriate threshold, which will be discussed later in this paper.

Unlike conventional cycle slip detection algorithms that use linear combinations of multiple frequency GPS observations, this cycle slip detection algorithm uses only single frequency GPS observations. Therefore, the proposed algorithm can detect cycle slips in any case regardless of sizes of simultaneous cycle slips in multiple frequencies. For example, ionospheric combination is widely used for cycle slip detection; however, this combination cannot detect cycle slips when the ratio of L1 and L2 cycle slips are almost the same as the ratio of wavelengths of L2 and L1. 


\subsection{Sensor error models}

In this proposed algorithm, a gyroscope and an odometer are used to estimate heading angle and distance traveled at the current epoch, respectively. However, the outputs of these two sensors contain errors, such as noise and bias, as well as the true values. In order to predict the effects of such errors on the MV, the errors of the MV need to be propagated and appropriate error models for these two sensors need to be applied. Some general error models have already been developed for these two sensors.

First, for the odometer sensor, Abbott ${ }^{19)}$ modeled the odometer output using the following equation.

$$
p(k)=\left(S_{\text {true }}+S_{\text {error }}\right) \cdot M+d_{k}-d_{k+1}
$$

In Eq. (3), two uniformly distributed random variables, $d_{k}$ and $d_{k+1}$ are defined between 0 and $S_{\text {true }}$. If $d_{k, k+1}=d_{k}-$ $d_{k+1}$ is defined, its mean and variance are 0 and $(1 / 6) \cdot S_{\text {true }}^{2}$ respectively. ${ }^{19)}$ In this algorithm, the time difference of the odometer output is needed to calculate the distance traveled, and it can be expressed as Eq. (4). The scale factor is related to the radius of the wheel of the vehicle, and it changes when the pressure in the tire of the wheel changes. For railway trains, it is reasonable to assume that the radius of wheels doesn't change over time. For cars, since, the pressure in the tire does not change abruptly, the scale factor can be assumed to change slowly over time.

$$
\delta p(k)=\left(S_{\text {true }}+S_{\text {error }}\right) \cdot \delta M+2 d_{k+1}-d_{k}-d_{k+2}
$$

The time difference of the odometer output contains three independent, uniformly distributed random variables, $2 d_{k+1}$, $d_{k}$ and $d_{k+2}$. The probability density function of a newly defined random variable, $g_{k}=2 d_{k+1}-d_{k}-d_{k+2}$, can be calculated using convolution integrals of the probability density functions of the three independent, uniformly distributed random variables. The result equations are shown below.

$$
f_{R}\left(g_{k}\right)=\left\{\begin{array}{c}
\left(1 / S_{\text {true }}\right) \cdot\left\{0.25 \cdot\left(g_{k} / S_{\text {true }}\right)^{2}+\left(g_{k} / S_{\text {true }}\right)+1\right\} \\
\left(-2 S_{\text {true }} \leq g_{k} \leq-S_{\text {true }}\right) \\
\left(1 / S_{\text {true }}\right) \cdot\left\{-0.25 \cdot\left(g_{k} / S_{\text {true }}\right)^{2}+0.5\right\} \\
\left(-S_{\text {true }} \leq g_{k} \leq S_{\text {true }}\right) \\
\left(1 / S_{\text {true }}\right) \cdot\left\{0.25 \cdot\left(g_{k} / S_{\text {true }}\right)^{2}-\left(g_{k} / S_{\text {true }}\right)+1\right\} \\
\left(S_{\text {true }} \leq g_{k} \leq 2 S_{\text {true }}\right)
\end{array}\right.
$$

Using Eq. (5), mean and variance of $g_{k}$ can be easily calculated as 0 and $(1 / 2) \cdot S_{\text {true }}^{2}$ respectively. Figure 1 shows the probability density function of the random variable, $g_{k}$ and the corresponding normal distribution. From Fig. 1, we now assume $g_{k}$ as a normally distributed random variable for further formulation.

The final error equation for the odometer sensor is shown below.

$$
(\delta p(k))_{\mathrm{error}}=S_{\mathrm{error}} \cdot \delta M+g_{k}
$$

In this paper, we don't consider odometer errors due to slippages of wheels.

Second, the error model of the MEMS gyroscope is well-

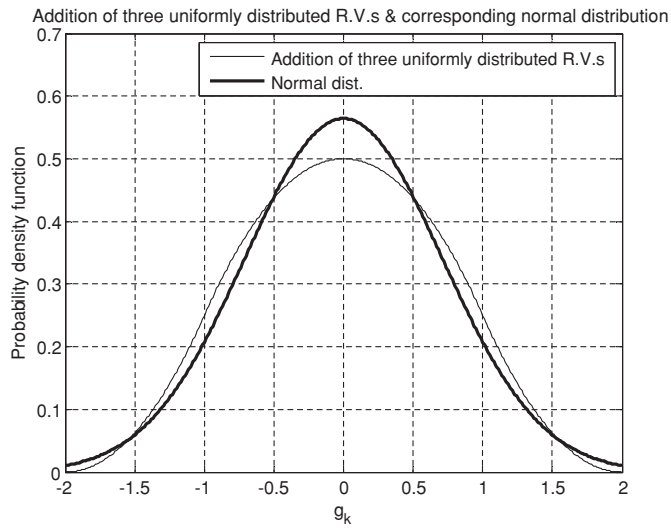

Fig. 1. Probability density function of the addition of three uniformly distributed random variables and corresponding normal distribution.

known as follows. ${ }^{20)}$

$$
\dot{\theta}_{\text {gyro }}(t)=\left(1+S_{f}\right) \dot{\theta}(t)+b(t)+\varepsilon(t)
$$

The bias error can be separated into a constant component and a random component. ${ }^{20)}$ It is assumed that the constant part of the bias error can be eliminated in the calibration process, and the resulting errors of the gyroscope include the random component of the bias stability error and the Gaussian noise. The bias stability can be modeled as a 1 st order Gauss-Markov process ${ }^{20}$ ) and it is assumed that the scale factor of the gyroscope is correctly estimated and compensated through calibration before using the gyroscope sensor. ${ }^{21)}$ Calibration is done by matching the output of the gyroscope to the known reference value. In addition, we assume that the misalignment error is also compensated before using the gyroscope sensor by alignment procedures. ${ }^{21,22)}$ Therefore, a simplified error model for the gyroscope can be expressed as follows.

$$
\dot{\theta}_{\text {gyro }}(t)=\dot{\theta}(t)+b(t)+\varepsilon(t)
$$

\subsection{Error propagation in monitoring value}

Now, the error equation is formulated by considering error terms of the two sensors introduced in section 2.2. The error equation helps to show the effect of the sensor errors manifested in the MV. First, the root mean square error of the monitoring value is expressed as follows.

$$
\operatorname{RMS}\{\operatorname{MV}(k)\}=\sqrt{\sigma_{\delta \Delta \nabla \phi}^{2}+\left|\delta \Delta \nabla D-\delta \Delta \nabla D_{\text {true }}\right|^{2}}
$$

Here, $\sigma_{\delta \Delta \nabla \phi}^{2}$ is the variance of the triple difference of carrier-phase measurement. Since the triple difference of ionospheric delay, tropospheric delay and ephemeris error are negligible their effects vanish in Eq. (9).

To further formulate the RMS error of the range related term, the following true and estimated vectors are defined. Figure 2 shows the simplified user and satellite configurations, indicating the distance related vectors.

$$
\begin{aligned}
& \hat{\vec{D}}_{\mathrm{U}}^{i}(k)=\hat{\vec{D}}_{\mathrm{U}}^{i}(k-1)+\delta \vec{D}^{i}(k)+\vec{p}_{\text {inertial }}(k) \\
& \vec{D}_{\mathrm{U}, \mathrm{t}}^{i}(k)=\vec{D}_{\mathrm{U}, \mathrm{t}}^{i}(k-1)+\delta \vec{D}^{i}(k)+\vec{p}_{\text {inertial }}(k)
\end{aligned}
$$




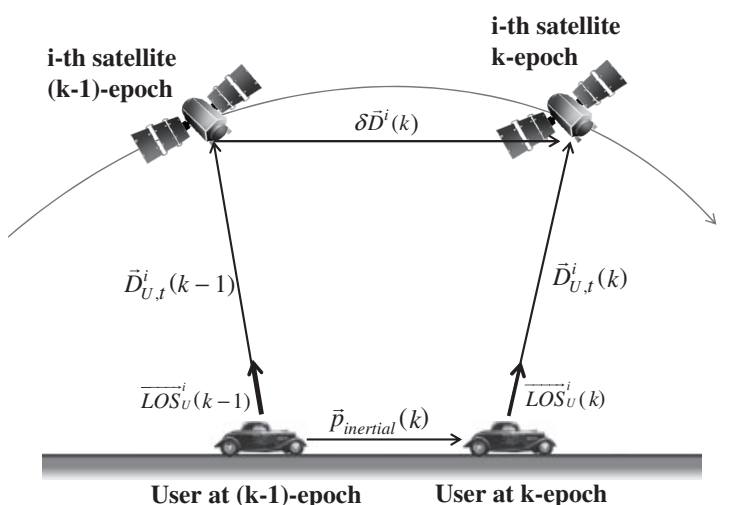

Fig. 2. User and satellite configuration during two epochs.

$$
\begin{aligned}
& \hat{\vec{D}}_{\mathrm{U}}^{i}(k-1)=\vec{D}_{\mathrm{U}, \mathrm{t}}^{i}(k-1)+\vec{D}_{\mathrm{U}, \text { error }}^{i}(k-1) \\
& \vec{p}_{\text {inertial }}(k)=\vec{p}_{\mathrm{t}, \text { inertial }}(k)+\vec{p}_{\text {error,inertial }}(k)
\end{aligned}
$$

Here, $\vec{p}_{\text {inertial }}(k)$ is the estimated displacement vector of the user between two successive epochs, and it consists of the true displacement vector, $\vec{p}_{\text {t.inertial }}(k)$, and the error vector, $\vec{p}_{\text {error,inertial }}(k)$, due to the errors in the gyroscope and odometer sensors. Other terms are explained in nomenclature.

Since the range terms related to the reference station can be completely eliminated, Eq. (9) can be rearranged as follows.

$$
\operatorname{RMS}(\mathrm{MV})=\sqrt{8 \cdot \sigma_{\phi}^{2}+\operatorname{RMS}\left(\nabla \hat{D}_{\mathrm{U}}(k)-\nabla \hat{D}_{\mathrm{U}}(k-1)\right)^{2}}
$$

In order to expand Eq. (11) further, user related range terms are defined as follows.

$$
\begin{aligned}
& \nabla \hat{D}_{\mathrm{U}}(k)=\hat{D}_{\mathrm{U}}^{i}(k)-\hat{D}_{\mathrm{U}}^{\mathrm{ref}}(k) \\
& =\hat{\vec{D}}_{\mathrm{U}}^{i}(k) \cdot \overline{\operatorname{LOS}}_{\mathrm{U}}^{i}(k) \\
& -\hat{\vec{D}}_{\mathrm{U}}^{\mathrm{ref}}(k) \cdot \overline{\operatorname{LOS}}_{\mathrm{U}}^{\mathrm{ref}}(k) \\
& \nabla D_{\mathrm{U}}(k-1)=D_{\mathrm{U}}^{i}(k-1)-D_{\mathrm{U}}^{\mathrm{ref}}(k-1) \\
& =\vec{D}_{\mathrm{U}}^{i}(k-1) \cdot \overline{\operatorname{LOS}}_{\mathrm{U}}^{i}(k-1) \\
& -\vec{D}_{\mathrm{U}}^{\mathrm{ref}}(k-1) \cdot \overline{\operatorname{LOS}}_{\mathrm{U}}^{\mathrm{ref}}(k-1) \\
& \nabla D_{\mathrm{U}, \mathrm{t}}(k)=D_{\mathrm{U}}^{i}(k)-D_{\mathrm{U}}^{\mathrm{ref}}(k) \\
& =\vec{D}_{\mathrm{U}, \mathrm{t}}^{i}(k) \cdot \overline{\operatorname{LOS}}_{\mathrm{U}}^{i}(k) \\
& -\vec{D}_{\mathrm{U}, \mathrm{t}}^{\mathrm{ref}}(k) \cdot \overline{\operatorname{LOS}}_{\mathrm{U}}^{\mathrm{ref}}(k)
\end{aligned}
$$

Using the definitions introduced in Eqs. (11) and (12), the second part in Eq. (11) can be further formulated and expressed as

$$
\begin{aligned}
\operatorname{RMS} & \left\{\nabla \hat{D}_{\mathrm{U}}(k)-\nabla \hat{D}_{\mathrm{U}}(k-1)\right\}^{2} \\
= & \mid\left\{\nabla \hat{D}_{\mathrm{U}}(k)-\nabla \hat{D}_{\mathrm{U}}(k-1)\right\} \\
& -\left.\left\{\nabla \hat{D}_{\mathrm{t}, \mathrm{U}}(k)-\nabla \hat{D}_{\mathrm{t}, \mathrm{U}}(k-1)\right\}\right|^{2} \\
= & \mid \vec{D}_{\mathrm{U}, \text { error }}^{i}(k-1) \cdot\{\overline{L O S} \\
& \left.-\overrightarrow{\mathrm{D}}_{\mathrm{U}, \text { error }}^{\text {ref }}(k)-1\right) \cdot\left\{\overline{L O S}_{\mathrm{U}}^{i}(k-1)\right\} \\
& +\left.\vec{p}_{\text {error,inertial }}(k) \cdot\left\{\overline{L O S}_{\mathrm{U}}^{i}(k)-\overline{L O S}_{\mathrm{U}}^{\mathrm{ref}}(k)\right\}\right|^{2}
\end{aligned}
$$

$$
\begin{aligned}
& \cong\left|\vec{p}_{\text {error,inertial }}(k) \cdot\left\{\overline{L O S}_{\mathrm{U}}^{i}(k)-\overline{\operatorname{LOS}}_{\mathrm{U}}^{\text {ref }}(k)\right\}\right|^{2} \\
& \leq\left|\vec{p}_{\text {error,inertial }}(k)\right|^{2} \cdot\left|\overline{L O S}_{\mathrm{U}}^{i}(k)-\overline{\operatorname{LOS}}_{\mathrm{U}}^{\mathrm{ref}}(k)\right|^{2} \\
& \leq\left|2 \cdot \vec{p}_{\text {error,inertial }}(k)\right|^{2} \\
& =4 \cdot\left|\vec{p}_{\text {error,inertial }}(k)\right|^{2}
\end{aligned}
$$

In Eq. (14), from the third to the fourth equality, the inner product of a change of line-of-sight vectors between two successive epochs and the error vector in the range direction induced by the user position error at the previous epoch can be assumed to be zero as follows.

$$
\begin{aligned}
& \vec{D}_{\mathrm{U}, \text { error }}^{i}(k-1) \cdot\left\{\overline{L O S}_{\mathrm{U}}^{i}(k)-\overline{\operatorname{LOS}}_{\mathrm{U}}^{i}(k-1)\right\} \\
& =\left(\vec{\varepsilon}_{\mathrm{U}}(k-1) \cdot \overline{\operatorname{LOS}}_{\mathrm{U}}^{i}(k-1)\right) \cdot \overline{\operatorname{LOS}}_{\mathrm{U}}^{i}(k-1) \\
& \cdot\left\{\overline{\operatorname{LOS}}_{\mathrm{U}}^{i}(k)-\overline{\operatorname{LOS}}_{\mathrm{U}}^{i}(k-1)\right\} \\
& =\left|\vec{\varepsilon}_{\mathrm{U}}(k-1)\right| \cos \eta^{i} \\
& \cdot\left\{\overline{L O S}_{\mathrm{U}}^{i}(k-1) \cdot \overline{\operatorname{LOS}}_{\mathrm{U}}^{i}(k)-1\right\} \\
& =\left|\vec{\varepsilon}_{\mathrm{U}}(k-1)\right| \cdot \cos \eta^{i}\left(\cos v^{i}-1\right) \\
& \approx 0
\end{aligned}
$$

The formulation is the same for the reference satellite as in Eq. (14). In the Eq. (14), $\eta^{i}$ is the angle between the error vector of user position and the line-of-sight vector of the $i$ th satellites at the previous epoch and $v^{i}$ is an angle between two successive line-of-sight vectors of the $i$ th satellite. Since $\nu^{i}$ is almost zero, the Eq. (14) also becomes zero. Therefore, it is shown that the user position error at the previous epoch to the $\mathrm{MV}$ is negligible.

Furthermore, from the fourth to the fifth equality, a worstcase scenario assumption is made in that the two line-ofsight vectors of the $i$ th and the reference satellites are parallel in opposite directions, as shown below.

$$
\begin{aligned}
& \left|\overline{\operatorname{LOS}}^{i}(k)-\overline{\operatorname{LOS}}^{\mathrm{ref}}(k)\right|^{2} \\
& =\left(\overline{\operatorname{LSS}}^{i}(k)-\overline{\operatorname{LOS}}^{\mathrm{ref}}(k)\right) \cdot\left(\overline{\operatorname{LOS}}^{i}(k)-\overline{\operatorname{LOS}}^{\mathrm{ref}}(k)\right) \\
& =\left|\overline{\operatorname{LSS}}^{i}(k)\right|^{2}-2\left|\overline{\operatorname{LSS}}^{i}(k)\right|\left|\overline{\operatorname{LOS}}^{\mathrm{ref}}(k)\right| \cos \varphi \\
& +\left|\overline{\operatorname{LOS}}^{\mathrm{ref}}(k)\right|^{2} \\
& \leq 4 \\
& \left(|\overline{L O S}|=1, \quad \varphi \text { is an angle between } \overline{\operatorname{LSS}}^{i}(k)\right. \\
& \text { and } \left.\overline{L O S}^{\text {ref }}(k)\right)
\end{aligned}
$$

As a result, the RMS error of the user related range can be expressed by an error vector in the displacement vector in Eq. (14). Now, this vector has to be related to the errors in the two sensors. The displacement vector of the user between the two epochs can be estimated by multiplying the distance traveled and the heading vector as in Eq. (16) by neglecting high order terms. They can be separated into the true and error values, and the heading vector is a unit vector. The epoch index $k$ is dropped for simplicity.

$$
\vec{p}_{\text {inertial }}=\delta p \cdot \vec{h}=\left\{\delta p_{\mathrm{t}}+\delta p_{\text {error }}\right\} \cdot\left(\vec{h}_{\mathrm{t}}+\vec{h}_{\text {error }}\right)
$$

In Eq. (16), $\delta p_{\text {error }}$ includes the odometer scale factor error and noise, and $\vec{h}_{\text {error }}$ is the integrated angle error induced by the noise and bias stability of the gyroscope. 
Therefore, the error vector in the displacement vector due to sensor errors can be formulated further as follows.

$$
\begin{aligned}
\left|\vec{p}_{\text {error,inertial }}\right| & =\left|\delta p_{\mathrm{t}} \cdot \vec{h}_{\text {error }}+\delta p_{\text {error }} \cdot \vec{h}_{\mathrm{t}}\right| \\
& \leq\left|\delta p_{\mathrm{t}}\right| \cdot\left|\vec{h}_{\text {error }}\right|+\left|\delta p_{\text {error }}\right| \cdot\left|\vec{h}_{\mathrm{t}}\right|
\end{aligned}
$$

The heading vector error and the integrated angle error need to be related to reflect the errors of the gyroscope from the displacement vector directly. Then, $\vec{h}_{\text {error }}$ can be calculated by rotating the true heading vector by $\theta_{\text {error }}$, which is the integrated angle error due to the gyroscope error, about the $U$-direction along the ENU coordinate. If the true heading vector is set as $\vec{h}_{\mathrm{t}}=\left(\begin{array}{lll}x & y & z\end{array}\right)^{\mathrm{T}}$, then the heading vector error can be estimated as follows.

$$
\begin{aligned}
\vec{h}_{\text {error }} & =\operatorname{Rot}\left(\theta_{\text {error }}, U\right) \vec{h}_{\mathrm{t}} \\
& =\left[\begin{array}{ccc}
\cos \theta_{\text {error }} & \sin \theta_{\text {error }} & 0 \\
-\sin \theta_{\text {error }} & \cos \theta_{\text {error }} & 0 \\
0 & 0 & 1
\end{array}\right] \vec{h}_{\mathrm{t}} \\
& =\left(\begin{array}{cc}
x \cos \theta_{\text {error }}+y \sin \theta_{\text {error }} \\
-x \sin \theta_{\text {error }}+y \cos \theta_{\text {error }} \\
z
\end{array}\right)
\end{aligned}
$$

where

$$
x^{2}+y^{2}+z^{2}=1 \quad\left(x^{2}+y^{2}=1 \text { for } 2 \mathrm{D} \text { case }\right)
$$

Since vehicles driven along roads are mainly constrained to a plane, 2D motion of a land vehicle user alone is considered here. Then, the norm of the heading vector error is $\sqrt{2 \cdot\left(1-\cos ^{2} \theta_{\text {error }}\right)}$. Using the fact that the norm of the heading vector is 1, Eq. (17) can be rearranged as follows.

$$
\left|\vec{p}_{\text {error,inertial }}\right| \leq\left|\delta p_{\mathrm{t}}\right| \cdot \sqrt{2 \cdot\left(1-\cos ^{2} \theta_{\text {error }}\right)}+\left|\delta p_{\text {error }}\right|
$$

We can express terms inside the square root in Eq. (19) simply by $\theta_{\text {error }}^{2}$, based on trigonometric relations and small angle assumption. By substituting Eq. (19) into Eqs. (11) and (14), the final error equation of the monitoring value can be expressed as

$\operatorname{RMS}(\mathrm{MV})=\sqrt{8 \cdot \sigma_{\phi}^{2}+4 \cdot\left\{\left|\delta p_{\mathrm{t}}\right| \cdot \theta_{\text {error }}+\left|\delta p_{\text {error }}\right|\right\}^{2}}$

With Eq. (20), by varying the range of the errors of the two sensors, corresponding errors in monitoring values can be predicted.

To make a conservative over-estimation of the contributions of the errors of the two sensors in the MV, $\left|\delta p_{\mathrm{t}}\right|$ is set to be the maximum distance traveled for heavy trucks ( $25 \mathrm{~m}$ for speed limit of $90 \mathrm{~km} / \mathrm{h}$ by law in South Korea) to maintain consistency with the simulation in the following section. The standard deviation of the L1 carrier-phases is set to $0.003 \mathrm{~m}$, which is larger than those for conventional receivers. Figure 3 shows the predicted MV errors for a range of 0 to $0.05 \mathrm{~m}$ and 0 to $5 \mathrm{deg} / \sqrt{\mathrm{hr}}$ for the odometer and gyroscope sensors, respectively.

Now, the previously predicted MV errors are consulted to select appropriate sensors that satisfy desired performance

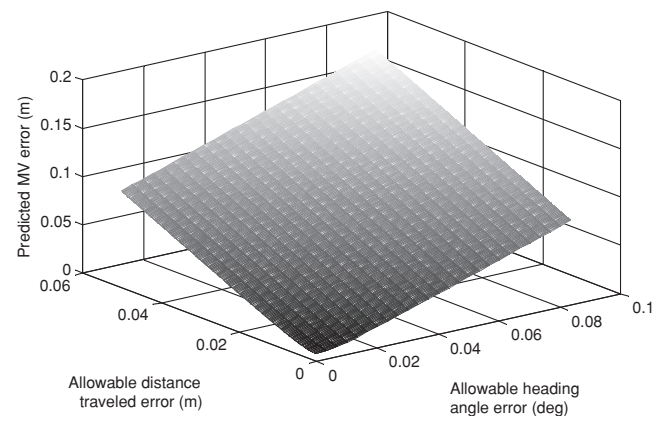

Fig. 3. Predicted MV errors for various errors for gyroscope and odometer sensors.

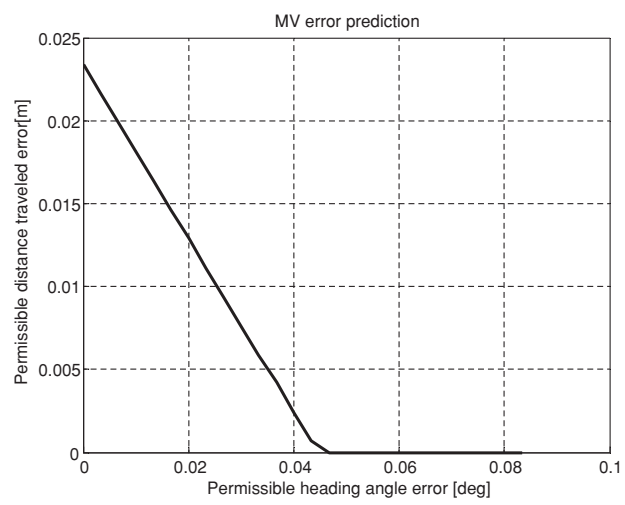

Fig. 4. Candidates of sensor error set whose MV error does not exceed half of the L1 wavelength for $95 \%$ probability.

parameters of the algorithm. In order to avoid degradation of the position accuracy due to cycle slips, the proposed algorithm should detect every cycle slip, with the smallest possible size of a cycle slip being \pm 1 cycle. Since a linear combination of dual-frequency carrier-phase measurements are not used, the sizes of cycle slips occurring directly reflect on the monitoring values, with some effects of the errors contributed by the two external sensors. We introduce a standard to find appropriate sensors to detect \pm 1 cycle slips as the smallest possible detectable size. From Fig. 3, every candidate of the sensor error sets is tested as to whether their RMS error of the MV exceeds half of the L1 wavelength with $95 \%$ probability. That is, a candidate from each sensor error set that does not exceed a quarter of the L1 wavelength is identified based on the relationship below.

$$
2 \cdot \operatorname{RMS}(\mathrm{MV})<\lambda_{1} / 2
$$

Equation (21) is set based on the miss detection probability introduced in section 2.4 and Table 2. Figure 4 shows the result of the possible candidates of sensor errors based on the standards described above.

It is clear that there are trade-offs in the relationship between the allowable sensor errors of the gyroscope and the odometer. Furthermore, any candidate set in the region bounded by the line, $x$ and $y$ axis is allowable, making errors in the MV less than a quarter of the L1 wavelength.

If we introduce defined error terms in section 2.2, errors in the distance traveled and the heading angle can be related to the sensor errors directly as follows. 


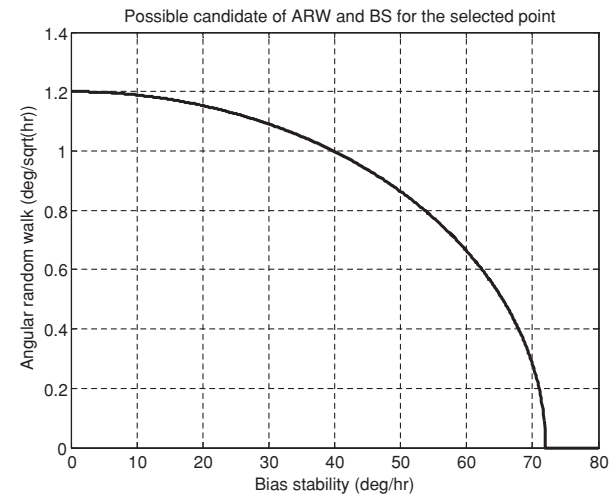

Fig. 5. Possible candidates of ARW and BS (points on and under the line) for a selected set of allowable errors from Fig. 4.

$$
\begin{aligned}
\theta_{\text {error }} & =\sqrt{\sigma_{\theta}^{2}+(b \cdot \Delta T)^{2}} \\
(\delta p)_{\text {error }} & =\sqrt{\sigma_{\delta p}^{2}+\left(\delta M \cdot S_{\text {error }}\right)^{2}} \\
& =\sqrt{\frac{1}{2} S_{\text {true }}^{2}+\left(\delta M \cdot S_{\text {error }}\right)^{2}}
\end{aligned}
$$

The heading angle error can be calculated by considering the noise of the estimated heading angle and the integrated bias stability error in the gyroscope. A distance error is induced by the noise contained in the difference of odometer output and the scale factor error. In general, the performance of the gyroscope is represented by the angle random walk and the bias stability that are related to the noise and bias in the gyroscope, respectively. The relationship between the standard deviation and the angle random walk is shown below, and the error due to the bias can be converted from the bias stability by a simple conversion. ${ }^{23,24)}$

$$
\begin{aligned}
\sigma_{\theta} & =\sqrt{f} \cdot \operatorname{ARW}(\mathrm{deg} / \sqrt{\mathrm{hr}}) \cdot(1 / \sqrt{3600}) \cdot \Delta T \\
b \cdot \Delta T & =\mathrm{BS}(\mathrm{deg} / \mathrm{hr}) \cdot(1 / 3600) \cdot \Delta T
\end{aligned}
$$

Here, $\Delta T$ is set to $1 \mathrm{~s}$ in this paper.

Again in Fig. 4, since the distance and the heading angle errors should be considered at the same time, a point must be chosen somewhere along the line to select appropriate sensors. Consider a case where the point corresponds to $(0.02 \mathrm{deg}, 0.0146 \mathrm{~m})$ for the heading angle and the distance errors, respectively. As before, if the scale factor error for the odometer is set at $0.05 \%$ of the true scale factor, then the possible noise of the odometer is approximately $0.0044 \mathrm{~m}$. The next figure shows the relationship between noise (expressed as the angle random walk) and bias (expressed as the bias stability) of the gyroscope, and their relationship can be expressed as a part of an ellipse, from Eqs. (22) and (23).

Since Eqs. (14) to (19) were formulated to over-estimate the errors in the MV induced from the sensor errors, the results shown above are conservative predictions. That is, possible candidates from the error equation would be included in the set of those determined from the simulation, which will be introduced in a later section.

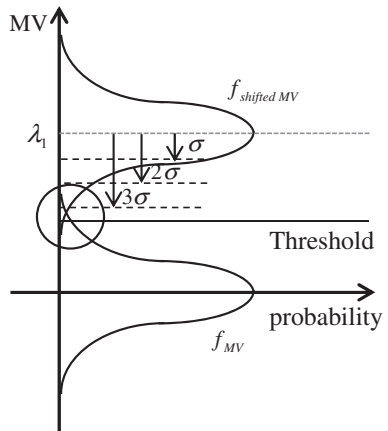

(a)

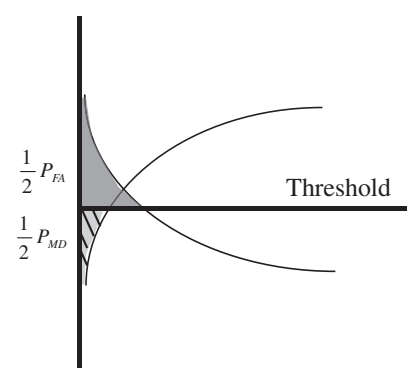

(b)
Fig. 6. Threshold determination scheme based on the Gaussian distribution. (a) Threshold based on the standard deviation of the probability density function of shifted MV. (b) Enlarged figure of the intersection of the probability density functions, MV and shifted MV (indicated as a circle in (a)).

\subsection{Threshold determination}

In order to validate whether the selected sensors can make cycle slip detection algorithm to detect 1 cycle slip as the smallest possible detectable cycle, we calculate the threshold for cycle slip detection. It is assumed that the MV has a normal distribution with zero mean and a standard deviation $\sigma$, and can be expressed as Eq. (24), along with a graph whose mean is 0 (Fig. 6(a)).

$$
\begin{aligned}
f_{\mathrm{MV}}(x \mid \mu, \sigma) & =(1 / \sqrt{2 \pi} \sigma) \exp \left\{-(x-\mu)^{2} /\left(2 \sigma^{2}\right)\right\} \\
& =(1 / \sqrt{2 \pi} \sigma) \exp \left\{-x^{2} /\left(2 \sigma^{2}\right)\right\}
\end{aligned}
$$

As mentioned before, since a cycle slip is an integer jump in the ambiguity, the smallest possible cycle slips are \pm 1 cycles. If a threshold that makes it possible for the proposed algorithm to detect such cycle slips is determined, other cycle slips whose absolute sizes are greater than 1 can be readily detected with that determined threshold. Since the MV when a cycle slip of -1 occurs has the same distribution as the MV that includes a +1 cycle slip. Therefore, the +1 cycle slip case alone is considered while determining the threshold here. When a +1 cycle slip occurs, the distribution of the noise shifts to have the mean of the L1 wavelength, and it is expressed as the following equation and a graph whose mean is the L1 wavelength (Fig. 6(a)).

$$
\begin{aligned}
f_{\text {shifted MV }}(x \mid \mu, \sigma) & =(1 / \sqrt{2 \pi} \sigma) \exp \left\{-(x-\mu)^{2} /\left(2 \sigma^{2}\right)\right\} \\
& =(1 / \sqrt{2 \pi} \sigma) \exp \left\{-\left(x-\lambda_{1}\right)^{2} /\left(2 \sigma^{2}\right)\right\}
\end{aligned}
$$

Consider a threshold, $T$, based on the standard deviation of the distribution, $f_{\text {shifted } \mathrm{MV}}$; the area bounded by the probability density function, $f_{\mathrm{MV}}$ and the threshold (the region shaded dark in Fig. 6(b) indicates the probability of the MV noise that is larger than the threshold. This probability is termed as "False Alarm" and data with this probability is sometimes detected as cycle slip even when cycle slip does not occur. On the other hand, the area bounded by the probability density function with mean $\lambda_{1}$ and the threshold (the region shaded light in Fig. 6(b) indicates the probability of 
the shifted MV noise that is smaller than the threshold. This probability is termed "Miss Detection" and some data with this probability cannot be detected even when cycle slip occurs. Both probabilities can be calculated using the following equations.

$$
\begin{aligned}
P_{\mathrm{FA}} & =2 \int_{T}^{\infty} f_{\mathrm{MV}}(x \mid \mu, \sigma) \mathrm{d} x \\
P_{\mathrm{MD}} & =2 \int_{-\infty}^{T} f_{\text {shifted } \mathrm{MV}}(x \mid \mu, \sigma) \mathrm{d} x
\end{aligned}
$$

The threshold, $T$, can be calculated based on the degree of reliability, $k$, with its mean using the following equation.

$$
\begin{aligned}
T & =f_{\text {Shifted MV }}{ }^{-1}(p \mid \mu, \sigma) \\
& =\left\{x: f_{\text {Shifted } \operatorname{MV}}(x \mid \mu, \sigma)=p\right\}
\end{aligned}
$$

where

$$
p=\int_{\lambda_{1}-k \cdot \sigma}^{\lambda_{1}+k \cdot \sigma} f_{\text {Shifted MV }}(x \mid \mu, \sigma) \mathrm{d} x
$$

An appropriate threshold among the calculated thresholds needs to be determined by considering the "False Alarm" and "Miss Detection" rates. For a conservative and safe detection of cycle slips, the "Miss Detection" limit is more important than the "False Alarm" limit; therefore more emphasis is given to the "Miss Detection" rate than the "False Alarm" rate, to determine the threshold.

\section{Simulation Results}

\subsection{Simulation environment}

In the previous section, the error equation for the monitoring value was formulated to detect cycle slips. In this section, data is simulated for GPS, gyroscope and odometer measurements to test the proposed algorithm, and to check the MV according to various sensor errors.

In order to reflect real measurements of GPS, GPS error sources are modeled, as described in Table $1 .{ }^{25}$ )

GPS simulation data are generated for approximately $100 \mathrm{~s}$ from 14:00, April 4th, 2012, and eight satellites (PRN 9, 12, 14, 15, 18, 21, 22 and 25) are observed during the simulation. Their elevation angles are approximately 60 , $43,24,36,81,20,45$ and $22 \mathrm{deg}$ respectively. PRN 18 is chosen to be the reference satellite because of its highest elevation angle. The distance between the initial position of the user and the reference station was approximately

Table 1. Simulation data generation strategy for GPS errors.

\begin{tabular}{ll}
\hline \multicolumn{1}{c}{ GPS errors } & \multicolumn{1}{c}{ Generation strategy } \\
\hline Ephemeris error & $\begin{array}{l}\text { Difference between satellite } \\
\text { positions based on precise orbit data } \\
\text { and navigation data }\end{array}$ \\
& IONEX and obliquity factor \\
Ionospheric delay & WAAS tropospheric delay model \\
Tropospheric delay & Modeled as an exponential function \\
Receiver noise & of elevation angle \\
& Neglect
\end{tabular}

$28 \mathrm{~km}$. A dynamic 5-ton truck is considered as the user with a $300 \mathrm{~kW}$ engine, and the acceleration and velocity profiles are generated using TruckSim $^{\circledR}$ software (provided by KARI, Korea Aerospace Research Institute). Fig. 7 shows the time history of the acceleration, the velocity and the trajectory of the truck.

Equation (3) was used to generate the odometer outputs with various scale factors ranging from 0 to $0.1 \mathrm{~m}$ with a $0.005 \mathrm{~m}$ step. The corresponding range of the odometer noise is calculated by multiplying the range of the scale factor by $\sqrt{1 / 6}$ because the odometer output contains a combination of two uniformly distributed random variables shown in section 2.2. According to this relationship, the odometer noise ranges from 0 to $0.041 \mathrm{~m}$ with about a $0.002 \mathrm{~m}$ step. Here, a scale factor error of $0.05 \%$ is assumed for comparison with the result in the previous section. It is expected that the odometer scale factor error could be larger than that as the result from the previous section was based on an overestimation strategy. Equation (7) is used to generate the gyroscope outputs for various gyroscope noises, and the bias stability of the gyroscope is fixed at $10 \mathrm{deg} / \mathrm{hr}$, to give more freedom to the ARW component, based on the result in Fig. 5. The bias stability is generated by modeling the Gauss-Markov process using the corresponding calculated

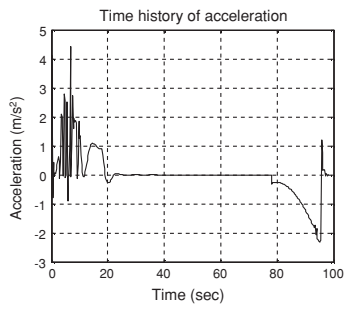

(a)

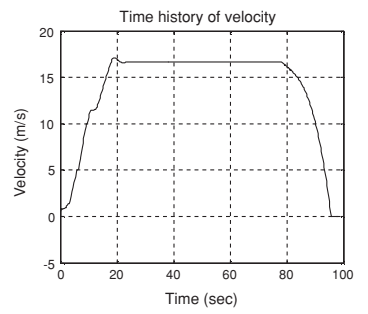

(b)

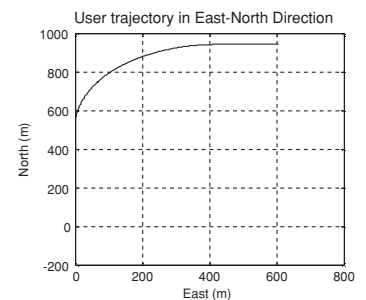

(c)

Fig. 7. Time history of acceleration (a) and velocity (b) of a truck user with a $300 \mathrm{~kW}$ engine generated by TruckSim software. User trajectory (c) is in the East-North coordinate.

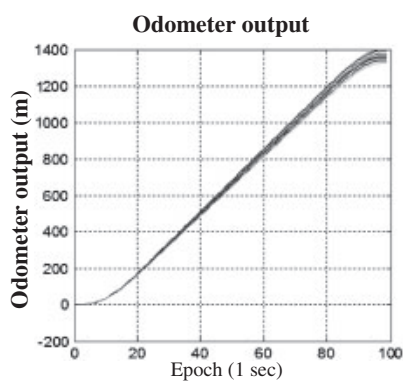

(a)

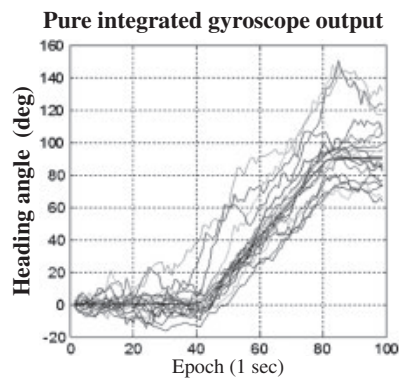

(b)
Fig. 8. Generated odometer output (a) and pure integrated gyroscope output (b) for various sizes of errors. 
standard deviation of the Gaussian noise in this model, and considering the length of the integration time. ${ }^{20)}$ Figure 8 shows the result of the generated odometer measurement and the pure integrated gyroscope outputs generated by the scheme described. It can be seen that only $0.05 \%$ of the scale factor error gives rise to approximately $2 \%$ distance error in the end.

\subsection{Simulation result}

Now the proposed algorithm is applied to the generated simulation data of GPS, the odometer and the gyroscope in the previous section. Since sensor measurements are generated for various sizes of the errors, the effects induced by these sensor errors can be seen by examining the monitoring values and determining whether sets with two sensors can be applied to determine \pm 1 cycle slips.

As in section 2.3, to determine the allowable sensor errors that make $\mathrm{MV}$ as small as possible to detect \pm 1 cycle slips, the RMS errors of MV are chosen for all visible satellites that are below a half of the L1 wavelength for $95 \%$ probability. Figure 9 shows the maximum allowable errors of two sensors that satisfy desired performance.

In Fig. 9, the candidates in the region below each line for each satellite satisfy the desired performance, described in Eq. (21), and the intersections of all regions are shaded. At a designated odometer scale factor and bias stability of the gyroscope, using a set of sensors in the shaded region enables the proposed algorithm to detect \pm 1 cycle slips. The thick line on the bottom left in the figure represents the predicted candidates from the theoretical approach in section 2.3 under designated bias stability of the gyroscope. As mentioned before in section 2.3, since the error equation is formulated to contain the maximum possible values of original sensor errors, the theoretical result lies within the result using simulation data. In conclusion, for the prediction of allowable sensor error limit, \pm 1 cycle slip can be detected by the proposed cycle slip detection algorithm when the odometer and gyroscope are used, whose errors are included in the shaded region for given scale factor error and bias stability for odometer and gyroscope respectively.

Now, consider a set of sensors having errors defined by the point marked with the cross in Fig. 9. This point is not

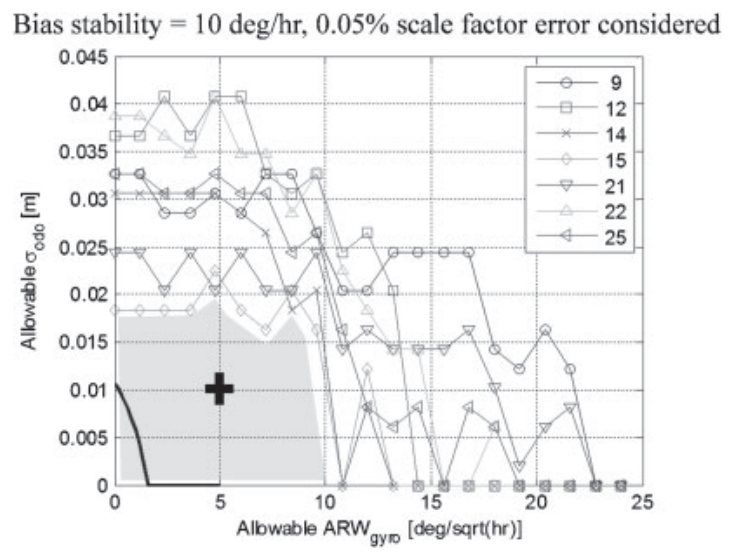

Fig. 9. Possible candidates of gyroscope and odometer errors. very conservative, and can cover all visible satellites at the same time. Figure 10 shows the time history of the monitoring values for all visible satellites colored in black, and also that when a \pm 1 cycle slip occurs, colored in gray. Based on the standard deviation of the shifted MV of the satellite with the lowest elevation angle, the threshold for a particular degree of reliability is also plotted in various types of lines. As mentioned before, a threshold of \pm 1 cycle slips are considered alone because they can readily detect cycle slips whose absolute sizes are greater than 1 cycle.

The thresholds, "Miss Detection" and "False Alarm" rates of the monitoring value shifted by +1 cycle are also indicated in Table 2. In this calculation of those two probabilities, two tails of the normal distribution are used.

From Fig. 10 and Table 2, the "Miss Detection" rates were too big for the cases with detection probability $95 \%$ and $99.7 \%$; thus, the thresholds of these cases are not suitable to detect cycle slip reliably. In the case of $5 \sigma$, its threshold missed only one cycle slip out of $1,000,000$ possible cycle slips; however, the alarms was activated too often even when there no cycle slips occurred; about 3 times out of 1,000 data. On the other hand, for the $4 \sigma$ case, it missed one cycle slip out of about 17,000 possible cycle slips, which is still fairly good. Moreover, it confused only one regular case for cycle slip out of 10,000 data.

In this paper, bias stability of the gyroscope and the scale factor error of the odometer are fixed. The angle random walk of the gyroscope can be fixed instead of the bias stabil-

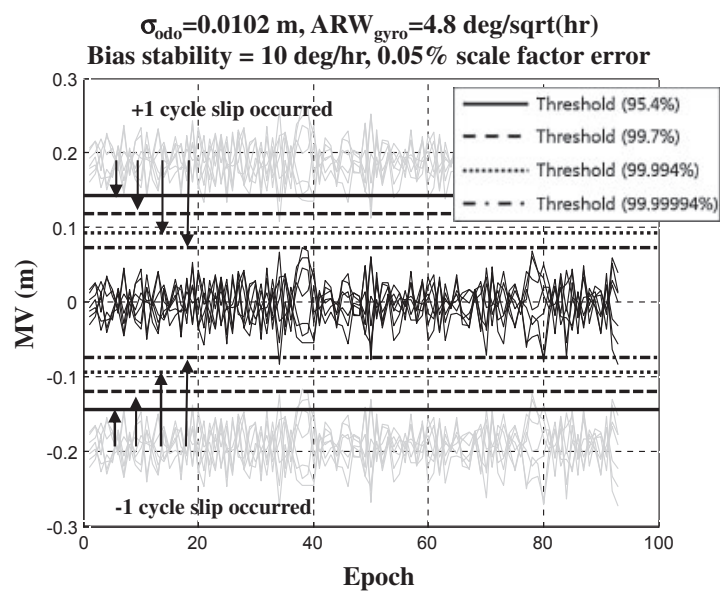

Fig. 10. Time history of MV (middle, black) and shifted MV with \pm 1 cycle slips $(+1$ cycle slip for the upper plot and -1 cycle slip for the lower plot) for all visible satellites.

Table 2. Threshold, miss detection and false alarm rates of the monitoring value shifted by +1 cycle.

\begin{tabular}{|c|c|c|c|c|}
\hline & \multicolumn{4}{|c|}{ Detection probability $(\%)$} \\
\hline & $95(2 \sigma)$ & $99.7(3 \sigma)$ & $99.994(4 \sigma)$ & $99.99994(5 \sigma)$ \\
\hline $\begin{array}{l}\text { Miss detection, } \\
P_{\mathrm{MD}}(\%)\end{array}$ & 5 & 0.3 & $6.33 \times 10^{-3}$ & $1.2 \times 10^{-4}$ \\
\hline $\begin{array}{c}\text { False alarm, } \\
P_{\mathrm{FA}}(\%)\end{array}$ & $3.14 \times 10^{-7}$ & $8.82 \times 10^{-5}$ & 0.010 & 0.25 \\
\hline Threshold (m) & \pm 0.1430 & \pm 0.1187 & \pm 0.0937 & \pm 0.0731 \\
\hline
\end{tabular}




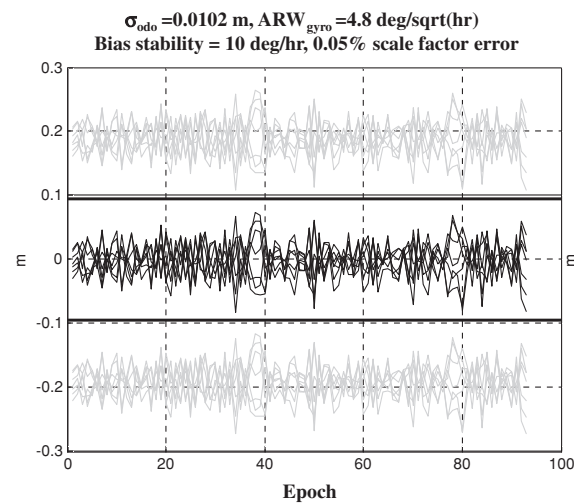

(a)

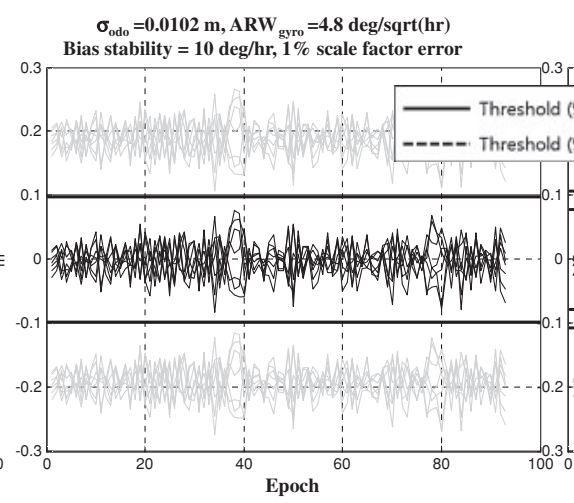

(b)

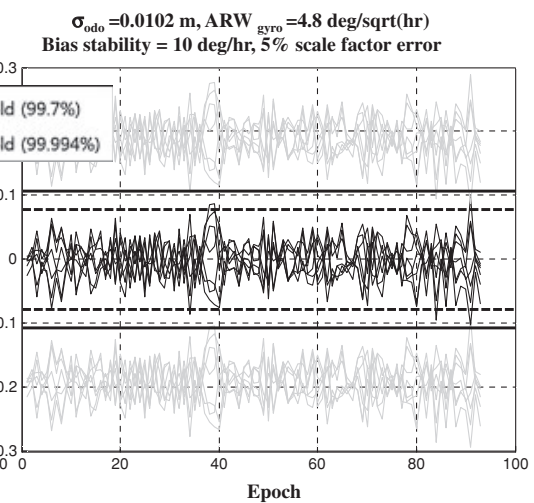

(c)

Fig. 11. Time history of MV (middle) and shifted MV (+1 cycle slip for the upper and -1 cycle slip for the lower) for all visible satellites with (a) $0.5 \%$, (b) $1 \%$, and (c) $5 \%$ odometer scale factor errors with $10 \mathrm{deg} / \mathrm{hr}$ bias stability, selected angle random walk of gyroscope and standard deviation of odometer.

ity, or both of the errors can be included in the RMS error of $\mathrm{MV}$, to see their effects at once. On the other hand, in the theoretical approach in section 2.3 , there were not enough margins for a scale factor error because the error equation is formulated to have exaggerated sensor errors. However, in the simulation procedure, the odometer scale factor error could be adjusted to predict the effect of the larger scale factor error in the MV. Figure 11 shows time histories of MV for all visible satellites for $0.5 \%, 5 \%$ and $10 \%$ odometer scale factor errors, with a $10 \mathrm{deg} / \mathrm{hr}$ bias stability for the gyroscope. Simulation results for $0.5 \%$ and $1 \%$ odometer scale factor errors detected all cycle slips occurring at every epoch when the $4 \sigma$ threshold is used. However, the $5 \%$ odometer scale factor error induced larger errors so that the $4 \sigma$ threshold confused normal MV values to cycle slips; and the $3 \sigma$ threshold missed some cycle slips. Since there are trade-offs among all sensor errors, sensors with higher performance to allow larger odometer scale factor error must be selected.

In Fig. 9, the maximum allowable sensor limits are different for all satellites since the amount of projected errors of sensors on observation domain is related to the satellite geometry. From section 2.3, the error of estimated distance at current epoch is incurred by the projected value of the estimation error of user displacement vector to the single differenced line-of-sight vector between $i$ th and reference satellites. Therefore, if the estimated user displacement vector is more aligned to the single differenced line-of-sight vector, the MV will be larger. In order to check this relationship, we plotted the MV with respect to the angle between single differenced line-of-sight vector and user displacement vector for all satellites together in Fig. 12.

Dots in the upper graph in Fig. 12 are the MV when the sensors having errors defined by the point of cross in Fig. 9. Crosses in the same figure are for the same gyroscope with odometer having standard deviation $0.0204 \mathrm{~m}$ and $\mathrm{x}$-shaped points in the lower figure in Fig. 12 are for the same odometer as the dot case with gyroscope having angle random walk of $10.8 \mathrm{deg} / \sqrt{\mathrm{hr}}$. The $\mathrm{MV}$ becomes larger when the angle between single differenced line-of-sight

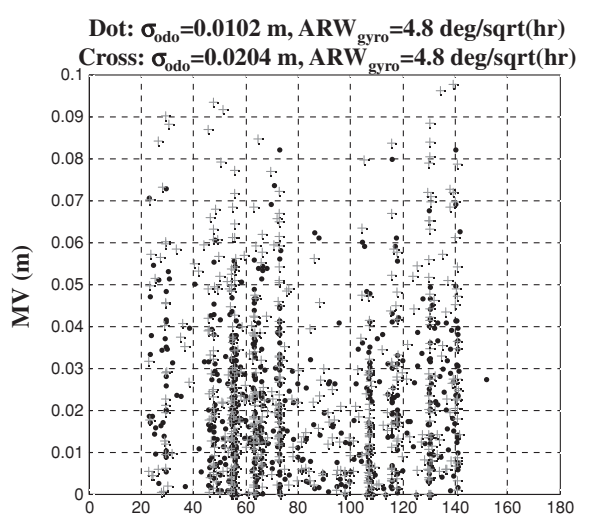

Angles between SD LOS and user displacement vector (deg)

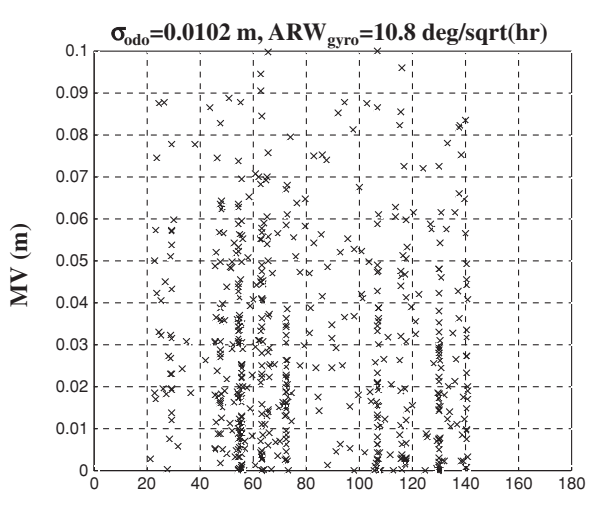

Angles between SD LOS and user displacement vector (deg)

Fig. 12. The MV for all satellites with respect to the angle between single differenced line-of-sight vector and user displacement vector.

and user displacement vector approaches 0 or $180 \mathrm{deg}$ for dots and crosses as expected. However, there is no such relationship for the $\mathrm{x}$-shaped case since the estimated heading angle is inaccurate to use because of a gyroscope with large angle random walk.

\section{Conclusion}

Cycle slip detection algorithms using inertial sensors, such as the one proposed in this paper can detect cycle slips in a single frequency GPS observation. Unlike the conventional cycle slip algorithms using linear combinations of 
GPS observations, single frequency based algorithms can detect cycle slips in any case regardless of sizes of simultaneous cycle slips in multiple frequencies.

In this paper, we also proposed a procedure for predictions of allowable sensor error limit for cycle slip detection using a theoretical approach. By deriving the error equation of the MV to detect cycle slips, the maximum allowable errors of the sensors to detect \pm 1 cycle slips could be predicted. In addition, allowable errors of the sensors using simulation data and the result included the candidates' prediction from the error equation. As a result, under a fixed bias stability and the odometer scale factor error, an odometer with noise whose standard deviation is approximately $0.0102 \mathrm{~m}$ and the gyroscope with $4.8 \mathrm{deg} / \sqrt{\mathrm{hr}}$ angle rate random walk were selected for the proposed algorithm to detect 1 cycle slip. This algorithm did not depend on positioning error at the previous epoch according to the theoretical analysis. The maximum allowable sensor error determined for each satellite was different from the others because the amount of projected errors of sensors on observation domain depends on the satellite geometry. Since errors from odometer sensors were partly originated from the change of radius of wheels, we expect that the odometer sensors can be used more reliably for railway trains than for cars.

The aiding sensors were confined to the odometer and the gyroscope in this paper. Currently, the predicted accuracy can be barely achieved by the odometer sensors that can be purchased in the market. Therefore, we are going to consider other IMU sensors, such as accelerometers for this research in the future. By replacing sensor error models according to the corresponding replaced sensors, the proposed error prediction approach can also be applied to other IMU sensors. This study is expected to provide a simple guide to the sensor selection before developing and operating systems under reasonable assumptions.

\section{Acknowledgments}

This research was supported by a grant from "Development of GNSS based Transportation Infrastructure Technology (06-A03)" funded by the Ministry of Land, Infrastructure and Transport of Korean government, contracted through SNU-IAMD at Seoul National University.

\section{References}

1) Pervan, B., Chan, F. C., Gebre-Egziabher, D., Pullen, S., Enge, P. and Colby, G.: Performance Analysis of Carrier-Phase DGPS Navigation for Shipboard Landing of Aircraft, Navigation: J. Inst. Navigation, 50 (2003), pp. 181-191.

2) van Bree, R. J. P., Buist, P. J. T. and Tiberius, C. C. J. M.: Lane Identification with Real Time Single Frequency Precise Point Positioning-A Kinematic Trial, Proc. 24th Int. Tech. Meeting of the Satellite Division of the Institute of Navigation (ION GNSS 2011), Portland, OR, 2011, pp. 314-323.

3) Goodliss, A., Manasseh, C. and Ekambaram, V.: Cooperative HighAccuracy Location (C-HALO) Service for Intelligent Transportation Systems: A Cost Benefit Study, Proc. 24th Int. Tech. Meeting of the Satellite Division of the Institute of Navigation (ION GNSS 2011), Portland, OR, 2011, pp. 2220-2232.
4) Blewitt, G.: An Automatic Editing Algorithm for GPS Data, Geophys. Res. Lett., 17 (1990), pp. 119-202.

5) Gao, Y. and McLellan, J. F.: An Analysis of GPS Positioning Accuracy and Reliability with Dual-Frequency Data, Proc. 9th Int. Tech. Meeting of the Satellite Division of the Institute of Navigation (ION GPS 1996), Kansas City, MO, 1996, pp. 945-951.

6) Gao, Y. and Li, Z.: Cycle Slip Detection and Ambiguity Resolution Algorithms for Dual-Frequency GPS Data Processing, Marine Geodesy, 22 (1999), pp. 169-181.

7) Neumann, J. B., Manz, A., Ford, T. J. and Mulyk, O.: Test Result from a New $2 \mathrm{~cm}$ Real Time Kinematic GPS Positioning System, Proc. ION GPS'96, Kansas City, MO, 1996, pp. 873-882.

8) Bisnath, S. B. and Langley, R. B.: Automated Cycle-Slip Correction of Dual-Frequency Kinematic GPS Data, Proc. 47th Annu. Conf., Canadian Aeronautics and Space Institute, Ottawa, Canada, 2000, pp. 121-125.

9) Dai, Z., Knedlik, S. and Loffeld, O.: Real-Time Cycle-Slip Detection and Determination for Multiple Frequency GNSS, Proc. 5th Workshop on Positioning, Navigation and Communication, Hannover, Germany, 2008, pp. 47-53.

10) Colombo, O. L., Bhapkar, U. V. and Evans, A. G.: Inertial-Aided Cycle-Slip Detection/Correction for Precise, Long-Baseline Kinematic GPS, Proc. 12th Int. Tech. Meeting of the Satellite Division of the Institute of Navigation (ION GPS 1999), Nashville, TN, 1999, pp. 1915-1922.

11) Altmayer, C.: Enhancing the Integrity of Integrated GPS/INS System by Cycle Slip Detection and Correction, Proc. IEEE Intelligent Vehicles Symp., Dearborn, MA, 2000, pp. 174-179.

12) Du, S.: An Inertial Aided Cycle Slip Detection and Identification Method for Integrated PPP GPS/MEMS IMU System, Proc. 24th Int. Tech. Meeting of the Satellite Division of the Institute of Navigation (ION GNSS 2011), Portland, OR, 2011, pp. 3183-3191.

13) Takasu, T. and Yasuda, A.: Cycle Slip Detection and Fixing by MEMS-IMU/GPS Integration for Mobile Environment RTK-GPS, Proc. 21st Int. Tech. Meeting of the Satellite Division of the Institute of Navigation (ION GNSS 2008), Savannah, GA, 2008, pp. 64-71.

14) Carlson, C. R., Gerdes, J. C. and Powell, J. D.: Error Sources When Land Vehicle Dead Reckoning with Differential Wheelspeeds, Navigation, 51 (2004), pp. 13-27.

15) $\mathrm{Yu}, \mathrm{M}$., Lee, J. and Park, H.: Comparison of SDINS In-Flight Alignment Using Equivalent Error Models, IEEE Trans. Aerospace Electronic Syst., 35 (1999), pp. 1046-1054.

16) Park, M. and Gao, Y.: Error and Performance Analysis of MEMSbased Inertial Sensors with a Low-cost GPS Receiver, Sensors, 8 (2008), pp. 2240-2261.

17) Song, J., Kim, Y., Park, B., Yun, H. and Kee, C.: Cycle Slip Detection Algorithm by Integrating Vehicle and Inertial Sensors for Land Vehicle Users, Int. Global Navigation Satellite Systems Society IGNSS Symp. 2011, Sydney, Australia, 2011.

18) Park, B.: A Study on Reducing Temporal and Spatial Decorrelation Effect in GNSS Augmentation System: Consideration of the Correction Message Standardization, Ph.D. Dissertation, Dept. Mech. and Aero. Eng., Seoul National University, Seoul, South Korea, 2007.

19) Abbott, E. C.: Land-Vehicle Navigation Systems: An Examination of the Influence of Individual Navigation Aids on System Performance, Ph.D. Dissertation, Dept. Mech. Eng., Stanford Univ., Stanford, CA, 1997.

20) Woodman, O. J.: An Introduction to Inertial Navigation, Comp. Lab., Univ. Cambridge, Cambridge, UK, 2007.

21) Titterton, D. H. and Weston, J. L.: Testing, Calibration and Compensation, Strapdown Inertial Navigation Technology, 2nd ed., The Institution of Electrical Engineers, Hertford, UK, 2004, pp. 223-242.

22) Nebot, E. and Durrant-Whyte, H.: Initial Calibration and Alignment of Low Cost Inertial Navigation Units for Land Vehicle Applications, J. Robotics Syst., 16 (1999), pp. 81-92.

23) Stockwell, W.: Angle Random Walk, Crossbow Technology, 2008, www.xbow.com

24) Stockwell, W.: Bias Stability Measurement: Allan Variance, Crossbow Technology, 2008, www.xbow.com

25) Yun, H., Yun, Y. and Kee, C.: Carrier Phase-based RAIM Algorithm Using a Gaussian Sum Filter, J. Navigation, 64 (2011), pp. 75-90. 